\title{
Estructura espacial y densidad de población en la ZMVM 1995-2010: evolución de un sistema urbano policéntrico
}

Iván Muñiz. Universitat Autònoma de Barcelona, Barcelona, España. Vania Sánchez. Universitat Autònoma de Barcelona, Barcelona, España. Miquel-Ángel García-López. Universitat Autònoma de Barcelona, Barcelona, España.

RESUMEN | En este trabajo se estima el efecto del policentrismo sobre la densidad de población en la Zona Metropolitana del Valle de México (ZMvM) entre 1995 y 2010. Los modelos teóricos policéntricos postulan que la densidad de la población debe caer a medida que la distancia a los centros de empleo aumenta. Los resultados confirman esta hipótesis a pesar de la fuerte inercia monocéntrica del área. Sin embargo, se advierte una creciente desconexión entre los espacios de empleo y los de vivienda, lo cual impediría reducir la distancia de los desplazamientos entre el lugar de residencia y el de trabajo. Un producto destacable del ejercicio llevado a cabo es que la capacidad estructurante que tiene la infraestructura viaria principal tiende a reforzarse en detrimento del efecto que ejercen los subcentros de empleo sobre la densidad de población.

PALABRAS ClAVE | centros urbanos, asentamientos humanos, distribución espacial.

ABSTRACT | This article estimates the effect of polycentrism on population distribution in the Metropolitan Zone of the Mexico Valley (MZMV) between 1995 and 2010. According to NUE [New Urban Economics] polycentric models, the higher the distance to employment centers, the lower the population density. It was found that sub-centers have a positive effect on population density, despite the strong monocentric inertia in the metropolitan area. However, the results also show an increasing gap between employment and housing spaces. This gap makes it increasingly difficult to reduce commuting distances. On the other hand, transportation infrastructure has a strong structuring capacity, and its effect on population density becomes stronger at the expense of the influence of employment sub-centers.

KEYWORDS | urban centers, human settlements, spatial distribution.

Recibido el 26 de febrero de 2013, aprobado el 7 de agosto de 2013

E-mail: ivan.muniz@uab.cat | vastrujillo@gmail.com | miquelangel.garcia@uab.cat

Correspondencia autor principal: Departament d'Economia Aplicada Edifici B Campus de la UAB, CP 08193, Barcelona, Espańa. 


\section{Introducción}

Diversos estudios han capturado el proceso de transformación de la estructura espacial del empleo que se ha dado en la Zona Metropolitana del Valle de México (zMvM en adelante). Su monocentrismo característico habría dado paso a un policentrismo (existencia de varios centros de empleo) que ha sido calificado como "incipiente" (Suárez \& Delgado, 2009). La investigación en torno al policentrismo de la ZMVM se ha centrado principalmente en la identificación y descripción de los subcentros de empleo (Graizbord \& Acuña, 2005; Aguilar \& Alvarado, 2005; Pradilla, 2005; Suárez \& Delgado, 2009; Casado, 2012), sin abordar su impacto sobre el precio del suelo, la densidad de empleo o de población, es decir, su capacidad estructurante.

El principal objetivo de este estudio es analizar el impacto de los subcentros sobre la densidad de población en la ZMvM durante el periodo 1995-2010. Como se ha demostrado en otros trabajos, el análisis del impacto de la estructura espacial del empleo sobre la densidad de población permite: a) poner a prueba las predicciones de los modelos teóricos de referencia; b) explicar las diferencias intrametropolitanas en el valor del suelo; c) prever el patrón de movilidad residencia-trabajo; y d) profundizar en el conocimiento de la naturaleza del policentrismo (McMillen \& Lester, 2003; Dowall \& Treffeisen, 1991; Muñiz, García-López \& Galindo, 2008).

El enfoque empírico utilizado se basa en la estimación de funciones policéntricas de densidad de población estáticas y dinámicas. En una función policéntrica de densidad, la densidad de población que se da en la zona $i$ de la ciudad $\left(D_{i}\right)$ se explica a partir de la distancia que la separa del CBD (centro de negocios, por sus siglas en inglés) y de los subcentros previamente identificados. Los resultados obtenidos (magnitud de los parámetros estimados, nivel de confianza, capacidad explicativa global) indican que: a) el policentrismo tiene un impacto limitado sobre la densidad de población en comparación con el СвD tradicional; b) la caída en la significatividad del gradiente de los subcentros podría avanzar una creciente desconexión entre el mercado de trabajo y de vivienda, con lo que el policentrismo no presentaría ventajas en términos de movilidad respecto del monocentrismo; y c) la ZMVM parece estar evolucionando desde un policentrismo reciente y limitado, hacia una estructura espacial tentacular donde los corredores metropolitanos pueden llegar a ejercer, en un futuro próximo, un papel vertebrador más relevante que el de los subcentros.

En lo que sigue, el artículo se organiza de la siguiente manera: en la sección 1 se pasa revista a la bibliografía reciente en torno al policentrismo; en la sección 2 se presentan la función policéntrica de densidad de población, los parámetros que se estimarán y los valores esperados; en la sección 3 se destacan las dinámicas recientes de población y empleo en las ciudades de América Latina, con especial atención al caso de la ZMVM; en la sección 4 se presentan las fuentes de los datos utilizados y se repasan algunas cuestiones metodológicas; en la sección 5 se describen los rasgos estilizados de la ZMVM; en la sección 6 se presentan los resultados obtenidos y, finamente, en la sección 7 se resumen las principales conclusiones del trabajo. 


\section{Policentrismo: ¿existe un único modelo?}

La tradicional concentración de puestos de trabajo en el centro de negocios de las grandes ciudades ha dado paso a un modelo de localización de la actividad económica más descentralizado, donde destaca la presencia de subcentros de empleo. El policentrismo parece haberse extendido a escala planetaria, afectando a ciudades de Norteamérica (Giuliano \& Small, 1991; McMillen \& McDonald, 1997; Shuckla \& Wadell, 1991; Shearmur \& Coffey, 2002), Europa (Muñiz et al., 2008; García-López \& Muñiz, 2010; Roca, Marmolejo \& Moix, 2009; Gilli, 2009; Veneri, 2010), Asia (Zheng, 1991), Oriente Medio (Alperovich \& Deutsch, 1994) y Latinoamérica (Dowall \& Treffeisen, 1991; Suárez \& Delgado, 2009; Rojas, Muñiz \& García-López, 2009; Aguilar \& Alvarado, 2005). Estos estudios muestran semejanzas, pero también notables diferencias, por lo que no parece adecuado presuponer la existencia de un solo modelo de policentrismo. Lo más probable es que coexistan diversas modalidades, atendiendo al origen de los subcentros y al impacto que generan sobre el precio del suelo, la densidad y la movilidad.

El peso del CBD. Mientras que en algunas ciudades el centro continúa aglutinando una parte relevante del empleo, en otras ha experimentado un retroceso que contrasta con el dinamismo observado en los subcentros de empleo o en las zonas más periféricas y menos densas. En algunas ciudades de EEUU -la mayoría localizadas en la Costa Este- el свD mantiene un peso relevante. Sin embargo, estas ciudades, antiguas y con un cierto aire europeo, no dejan de ser una excepción. En la actualidad, el peso del centro en términos de empleo en las ciudades norteamericanas es muy limitado (Lee, 2007; Meijers \& Burger, 2009). En contraste con las dinámicas observadas en buena parte del territorio norteamericano, en Europa, Asia y Latinoamérica la descentralización del empleo parece haber sido más limitada. También han emergido subcentros y se han dado procesos de localización dispersa, pero aun así, los centros históricos de sus grandes ciudades han mantenido un porcentaje de empleo muy por encima de los estándares norteamericanos (Muñiz, Galindo \& García-López, 2003; Bertaud, 2004; Gilli, 2009).

El argumento más socorrido para explicar el importante porcentaje de puestos de trabajo que sigue reteniendo el centro histórico de algunas ciudades es la existencia de restricciones a la movilidad (elevados costes de transporte). Una explicación alternativa pasa por destacar la importancia inercial de las economías de aglomeración, así como la dotación de servicios culturales y sociales orientados hacia el consumidor que se dan en el centro histórico (Glaeser \& Gottlieb, 2009). La capacidad para generar economías de aglomeración en el CBD no solo afecta a la concentración del empleo, sino que también determina el patrón de movilidad residencia-trabajo y condiciona la localización de los subcentros, que tienden a situarse cerca del CBD (Ahfeldt \& Wendland, 2013).

Origen. El policentrismo puede surgir a partir de la descentralización reciente del empleo, o bien de la integración funcional de centros de menor tamaño. Al primer tipo de policentrismo lo denominamos policentrismo descentralizado y al segundo, 
policentrismo christalleriano. Los subcentros descentralizados han recibido en EEUU el nombre de edge cities, destacando su carácter novedoso respecto de otras concentraciones periféricas de empleo que ya habían aparecido con anterioridad. Los subcentros christallerianos -también calificados como centros regionales de segundo orden - habrían ofrecido en el pasado servicios menos especializados que el CBD, destinados a cubrir las necesidades de la población de sus alrededores (Muñiz et al., 2003, 2008; Champion, 2001; Phelps \& Parsons, 2003).

Movilidad residencia-trabajo. La evidencia empírica no permite afirmar, pero tampoco negar de forma categórica, que el policentrismo represente mejora alguna en términos de movilidad respecto del monocentrismo (Aguilera, 2005; Veneri, 2010). Para algunos autores, el policentrismo permitiría mejorar la colocalización de empleos y trabajadores y, como resultado, reducir la distancia de los desplazamientos entre el lugar de residencia y el de trabajo (Gordon, Richardson \& Wong, 1986; Cervero \& Wu, 1998). El centro tradicional seguiría siendo el principal destino de los desplazamientos exteriores, pero los subcentros recibirían trabajadores procedentes de localidades próximas. La distancia promedio de los desplazamientos residencia-trabajo se reduciría a partir de dos mecanismos: a) unos centros autocontenidos con capacidad para ofrecer empleo a los trabajadores residentes (aumento del job ratio), y b) la posibilidad de contar con un subcentro próximo como destino alternativo al CBD tradicional para los desplazamientos externos. Este modelo de movilidad asociado a una estructura policéntrica, calificado en Bertaud (2004) como "urban village" es, a grandes rasgos, el mismo que se desprende de la mayoría de modelos policéntricos de naturaleza exógena de la Economía y de la Geografía Urbana (Sullivan, 1986; Wieand, 1987; White, 1999).

Autores como Giuliano y Small (1991) sostienen, por el contrario, que el policentrismo solo sería más eficiente que el monocentrismo si los individuos decidieran su lugar de residencia teniendo en cuenta de forma preferente la distancia al lugar de trabajo del jefe de familia. Sin embargo, si el lugar de residencia se escoge teniendo en cuenta otros factores, como el contacto con la naturaleza o la calidad de las escuelas locales; y si además trabaja más de un miembro de la familia, todo ello en un contexto de costes de transporte decrecientes y aumento de la renta per cápita, entonces el policentrismo vendría acompañado de flujos cada vez más abiertos y difícilmente predecibles.

Transitoriedad y capacidad estructurante (Efectos dinámicos). Otra de las cuestiones no resueltas en torno al policentrismo es si se trata de un modelo espacial estable o de un modelo transitorio. Algunos autores sostienen que el policentrismo podría ser un equilibrio espacial a medio camino entre el monocentrismo y la total dispersión. Gordon y Richardson (1996) plantearon esta hipótesis como posible explicación para la caída observada en el porcentaje de empleo concentrado en los centros de empleo de Los Ángeles (eEUu). Según dichos autores, la caída en los costes de transporte y las mejoras en el terreno de las telecomunicaciones habrían permitido dilatar el ámbito espacial de las economías de aglomeración, reduciendo con ello los costes de congestión. A pesar de las voces críticas que se han alzado contra el excesivo determinismo 
tecnológico del argumento (Boderman, 1998; Shearmur \& Coffey, 2002; Muñiz \& García-López, 2009; Glaeser \& Gottlieb, 2009), en un buen número de trabajos se han obtenido resultados similares en otras ciudades de EEUu, Europa y América Latina (Giuliano \& Redfearn, 2005; García-López \& Muñiz, 2010; Gallo, Garrido \& Vivar, 2010; Pfister, Freestone \& Murphy, 2000; Lee, 2007; Gilli, 2009).

Algunos autores han señalado la posibilidad de que los subcentros distribuidos a lo largo de una infraestructura viaria acaben constituyendo corredores lineales de actividad. En tal caso, el policentrismo sería una estructura espacial transitoria entre el monocentrismo y un modelo tentacular, donde la actividad económica se localizaría preferentemente en el CBD y en los principales corredores metropolitanos (Craig \& Kohlhase, 2010).

En cuanto a la capacidad estructurante de los subcentros, diferentes estudios han detectado la existencia de gradientes no significativos o con el signo contrario al esperado (McMillen \& Lester, 2003; Dowall \& Treffeisen, 1991), al estimar funciones policéntricas de densidad de población. Otro grupo de trabajos ha dado cuenta de la limitada capacidad de los subcentros para afectar la movilidad de la población cercana (Giuliano, 1995; Handy, 1996; Weber, 2003). Tomados en conjunto, estos resultados parecen indicar que los subcentros tienen una capacidad estructurante o vertebradora cada vez más limitada (Muñiz \& García-López, 2012).

A modo de resumen, podemos concluir que: a) no existe un solo modelo de policentrismo; b) las diferencias pueden deberse a factores tan variados como el peso histórico del centro, los costes de transporte vigentes, la existencia de un sistema urbano regional preindustrial, o la implantación de nuevas tecnologías en el proceso de producción; y c) la amplia variedad de modelos policéntricos observados suele venir acompañada de impactos diferenciados sobre aspectos tan relevantes para la planificación urbana como el valor del suelo o la movilidad residencia-trabajo.

\section{La función policéntrica de densidad de población}

Los modelos de ciudad monocéntrica (Alonso, 1960; Muth, 1961; Mills, 1967) aparecidos durante las décadas de los sesenta y setenta describían una ciudad ideal donde un porcentaje importante del empleo se localizaba en el centro (CBD), y tanto el precio del suelo como la densidad poblacional se fijaban mediante un mecanismo compensador según el cual los mayores costes de transporte que soportaban los trabajadores residentes en localizaciones alejadas del centro debían ser compensados con una renta del suelo menor. Tres de las principales predicciones de estos modelos son: a) el precio cae al aumentar la distancia al CBD; b) los desplazamientos externos residencia-trabajo tienen su origen en la periferia y su destino en el CBD; у с) la densidad de población debe decrecer suavemente al aumentar la distancia al свD (Gráfico 1). Estas predicciones coincidían en gran medida con lo observado para un buen número de ciudades de diversos países y continentes, lo cual dotó de gran credibilidad al modelo.

Debido a que no siempre resulta sencillo obtener información sobre precios del suelo y flujos de movilidad, la validación del modelo teórico se ha llevado a cabo principalmente por medio del análisis del comportamiento de la densidad de población. Otra razón que explica el interés que despierta dicha variable es que 
puede modificarse por medio de la planificación urbana cuando se observan o prevén problemas asociados a su exceso (congestión) o defecto (dispersión).

La función de densidad más comúnmente utilizada para caracterizar una estructura espacial monocéntrica es la Función de Densidad Exponencial Negativa. Conociendo la densidad de población de cada zona de la ciudad y a qué distancia se encuentra del $\mathrm{CBD}$, es posible estimar los dos parámetros de esta función: la densidad teórica central $\left(y_{1}\right)$ y el gradiente de densidad $\left(y_{2}\right)^{1}$ (Ecuación 1).

Ecuación 1. Función de densidad exponencial negativa $\ln D_{i}=y_{1}+y_{2} D I S T_{C B D_{i}}+\epsilon_{i}$

\section{GRÁfICO 1 | Función de densidad ciudad monocéntrica}

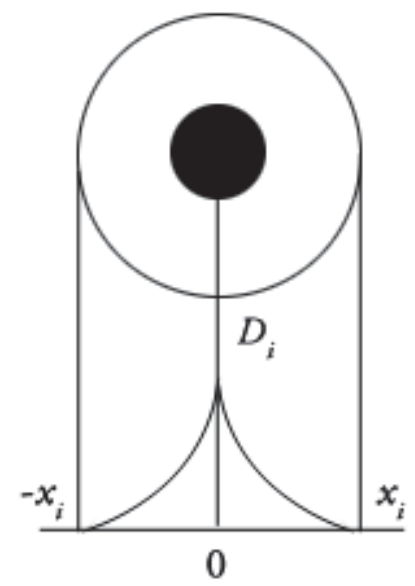

FUENTE ELABORACIÓN PROPIA

La función de densidad policéntrica estándar, la función multiplicativa, incorpora como variables explicativas tantas distancias como centros de empleo. Por tanto, se estima un número de gradientes de densidad equivalente al número de centros de empleo identificados (Dowall \& Treffeisen, 1991; García-López, 2006; Muñiz et al., 2008). Los modelos policéntricos de la Economía y Geografía Urbana (White, 1999), sustento teórico de dicha función, predicen una caída global en la distancia recorrida en los desplazamientos residencia-trabajo, debido a que los trabajadores que residen en la periferia suburbana tendrán como posible destino, además del $\mathrm{CBD}$, un subcentro de empleo más cercano. Como resultado, las zonas más próximas a los subcentros de empleo serán más valoradas, pues conllevan menores costes de movilidad. Se espera, por tanto, que los gradientes estimados para los subcentros tengan un signo negativo, replicando -a menor escala espacial- el efecto que ejerce la distancia al CBD (Gráfico 2).

$1 \quad$ El gradiente de densidad de una función de densidad monocéntrica puede ser interpretado como la "pendiente" de la función exponencial negativa. El gradiente representa, de hecho, un valor constante para cualquier distancia al centro (condición que no cumple la pendiente) y mide la variación proporcional de la densidad ante un incremento marginal de la distancia. Se trata, por tanto, de un parámetro a medio camino entre una pendiente y una elasticidad. 
Proximidad al CBD, origen, movilidad y transitoriedad: efectos esperados sobre los parámetros de una función de densidad policéntrica de población

Proximidad al CBD. Si los subcentros se localizan a poca distancia del CBD, los parámetros estimados se verán afectados tanto en su valor, como en su significatividad estadística. El principal problema es que las áreas de influencia del СвD y de los subcentros se solaparían, con lo cual alejarse de un subcentro podría suponer acercarse al CBD, lo que conllevaría que la densidad no cayera al aumentar la distancia al subcentro, sino que aumentara. Como resultado, los gradientes estimados pueden resultar no significativos o incluso presentar valores positivos (Muñiz et al., 2008).

Origen. Los subcentros de origen christalleriano han ejercido un importante papel vertebrador sobre el territorio, afectando el valor del suelo y la densidad durante décadas, o siglos en algunos casos. De hecho, los subcentros christallerianos son antiguos sistemas monocéntricos de segundo orden que, a pesar de integrarse en una región urbana y, por tanto, abrir sus mercados de trabajo, siguen presentando una cierta autocontención, afectando las condiciones de densidad de forma similar a como lo hacían en el pasado. Por consiguiente, ceteris paribus, es de esperar la obtención de gradientes con mayor valor y significatividad para los subcentros christallerianos que para los descentralizados (Muńiz et al., 2008).

Ecuación 2. Función de densidad exponencial multiplicativa $\ln D_{i}=y_{1}+y_{2} D I S T_{C B D_{i}}+\sum_{n=1}^{N} \beta_{n i}+\epsilon_{i}$

GRÁfICo 2 | Densidad y distancia al centro. Modelo policéntrico

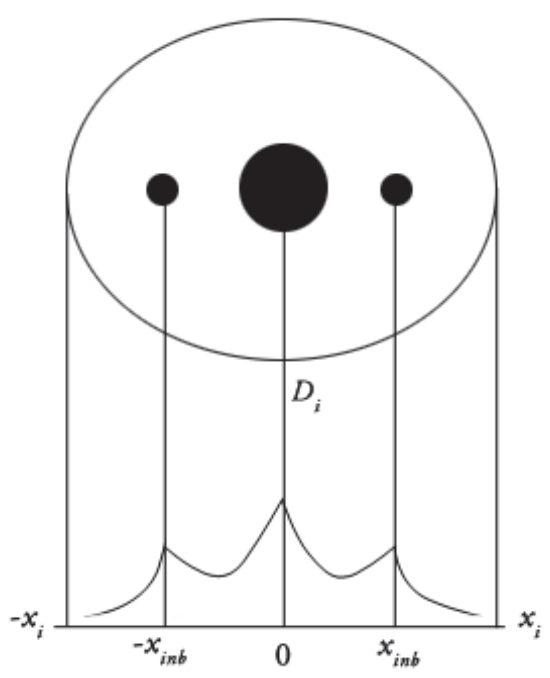

FUENTE ELABORACIÓN PROPIA 
Movilidad residencia-trabajo. El policentrismo del tipo urban village comporta una reducción en las distancias recorridas para los desplazamientos con origen en la periferia, en comparación con el monocéntrico. Por el contrario, el policentrismo calificado por Bertaud (2004) como de movimientos aleatorios se caracteriza por ser un patrón de movilidad más abierto y menos predecible. Ambos modelos llevan asociadas diferentes valoraciones para el suelo que rodea los subcentros, lo cual determina su intensidad de uso (densidad). Es de esperar, por consiguiente, que el valor y la significatividad de los gradientes de densidad estimados para los subcentros christallerianos sean mayores que para el caso de los subcentros descentralizados.

Transitoriedad y desestructuración (Efectos dinámicos). Si un porcentaje creciente del empleo se descentraliza desde el centro y los subcentros hacia la periferia adoptando un patrón de localización cada vez más disperso, debiera observarse una caída en el valor de los gradientes. Si además los subcentros afectan cada vez menos la densidad de población, generando un sistema urbano crecientemente desestructurado, debiera obtenerse unos gradientes cada vez menos significativos y la capacidad explicativa global del modelo $\left(\mathrm{R}^{2}\right)$ debería caer. Por otro lado, si el policentrismo que se da a lo largo de una infraestructura viaria evoluciona hasta convertirse en un corredor de empleo, y en lugar de utilizar como referencia una estructura espacial de tipo tentacular se utiliza una policéntrica, los parámetros estimados estarían cada vez más sesgados, la significatividad de los gradientes se reduciría y la capacidad explicativa global del modelo se resentiría.

\section{Dinámicas de población y empleo en las grandes ciudades de América Latina}

Las ciudades latinoamericanas no han quedado al margen de los intensos cambios que se han dado en el patrón de localización de la población y del empleo durante las últimas décadas. En primer lugar, cabe señalar la aceleración en el consumo de suelo urbanizado. El término dispersión urbana (urban sprawl), utilizado para caracterizar el modelo de crecimiento de las ciudades de EeuU y Europa, posiblemente no capture correctamente la dinámica espacial de las metrópolis latinoamericanas, debido a que el aumento del suelo urbanizado viene generalmente acompañado de incremento poblacional ${ }^{2}$. Aun así, todo parece indicar que el ritmo de crecimiento en el consumo de suelo urbanizado es muy superior al de la población, debido principalmente a la proliferación de áreas residenciales de baja densidad en la periferia suburbana (Arellano \& Roca, 2010; Heinriechs, Nuissl \& Rodríguez, 2008; Aguilar, 2002; Lungo, 2000; Abramo, 2011; Monkkonen, 2011), alimentando con ello un modelo

2 En Europa y eEuu, la dispersión urbana se caracteriza a partir de elementos morfológicos (consumo de suelo, fraccionamiento del espacio, densidad residencial decreciente), en un contexto de estabilidad demográfica y clara descentralización de la población y del empleo (Muñiz \& GarcíaLópez, 2013). En el caso de las ciudades latinoamericanas, aunque los cambios morfológicos experimentados son similares, el contexto es diferente: siguen creciendo en términos demográficos y el centro principal retiene un porcentaje relevante de población y empleo. 
espacial crecientemente fragmentado y discontinuo (Janoschka, 2002; Abramo, 2011; De Mattos, 1999; Ojima \& Hogan., 2009).

En segundo lugar, el proceso de expansión física de las ciudades ha supuesto la reorganización espacial de la población y del empleo. Si bien es cierto que la vida en las grandes áreas metropolitanas latinoamericanas sigue orbitando por lo general alrededor del centro tradicional, el peso de este parece haber caído tanto en términos de población como de empleo. Durante las últimas dos décadas, el impulso descentralizador ha afectado con especial intensidad al empleo (UN Habitat, 2012; Roitman \& Phelps, 2010; Arellano \& Roca, 2010; Rubiera \& Aponte, 2009; Ludeña, 2006; Borsdorf, 2003; Lungo, 2000; De Mattos, 1999).

Por último, son numerosos los trabajos que han identificado subcentros de empleo para ciudades concretas: zMvM, México (Aguilar \& Hernández, 2011); Concepción, Chile (Rojas et al., 2009); Santiago, Chile (Becerril-Padua, 2000); Lima, Perú (Gonzales de Olarte \& Del Pozo Segura, 2012); Bogotá, Colombia (Dowall \& Treffeisen, 1991; Rubiera \& Aponte, 2009; Avendaño, 2013); Buenos Aires, Argentina (Vecslir \& Ciccolella, 2012) ${ }^{3}$, etcétera.

\section{Policentrismo en la ZMvM}

Los estudios previos sobre la estructura espacial de la ZMVM detectan indicios de policentrismo, aunque también seńalan el importante peso que sigue manteniendo el área central. La relevancia del centro histórico se expresaría de diferentes formas. Por un lado, el porcentaje de empleos que concentra seguiría siendo muy relevante (Suárez \& Delgado, 2009; Aguilar \& Alvarado, 2005). Por otro, el área central es el origen y sobre todo el destino de la mayoría de los desplazamientos residenciatrabajo (Graizbord \& Acuńa, 2005; Nava, 2010). Por último, una buena parte de los subcentros de empleo se encuentran próximos al СвD tradicional (Pradilla, 2005; Aguilar \& Alvarado, 2005; Aguilar \& Hernández, 2011).

Las técnicas utilizadas para identificar los subcentros de la ZMVM han sido muy variadas: un umbral fijo de empleo (Aguilar \& Alvarado, 2005); una combinación de umbrales fijos aplicados al volumen de empleo, a la densidad de empleo y al jobratio (Casado, 2012); umbrales estadísticos para el volumen de empleo y el porcentaje de empleo en el sector terciario (Aguilar \& Hernández, 2011); un umbral para el job-ratio combinado con la captura de residuos significativos, tomando como referencia una función de densidad monocéntrica de empleo (Suárez \& Delgado, 2009); o bien la metodología de flujos sobresalientes (Graizbord \& Acuña, 2005). La amplitud de estudios dedicados a la identificación de subcentros ha dado como resultado un listado que varía significativamente, dependiendo de la técnica adoptada. Aun así, más allá de la frontera del área central, la lista de subcentros identificados resulta bastante estable (Gráfico 3). 
GRÁFICo 3 | Resultados de estudios previos de subcentros de México

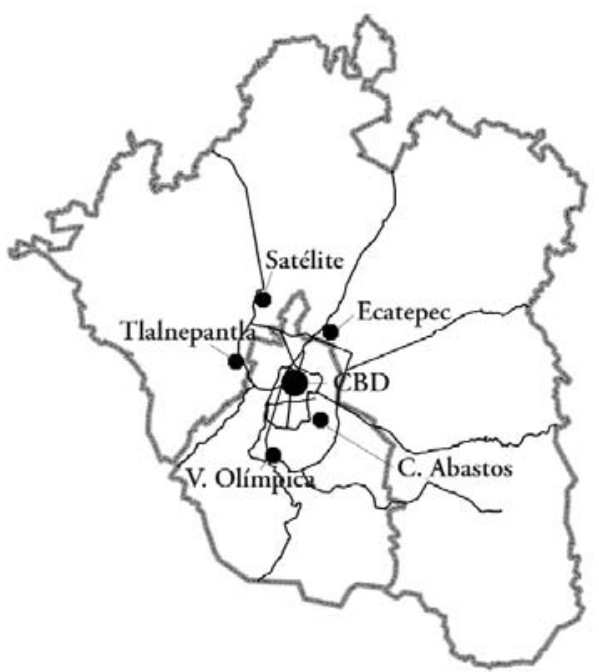

Casado, 2012

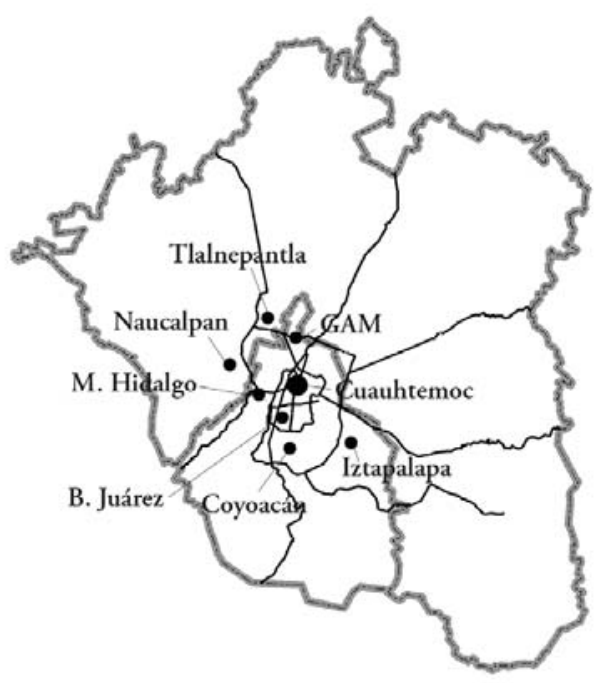

Graizbord y Acuńa, 2005

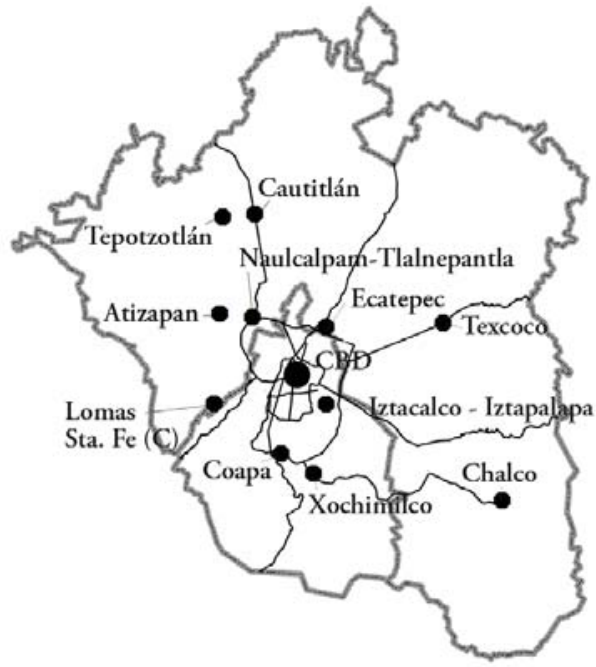

Suárez y Delgado, 2009

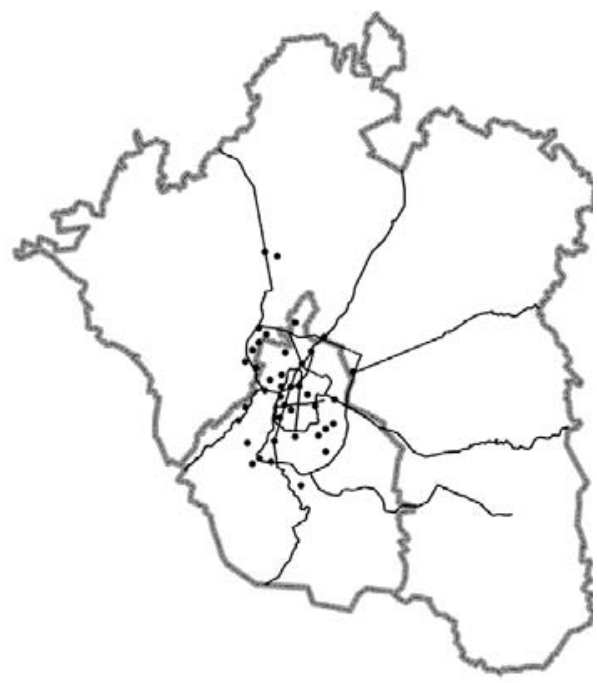

Aguilar y Hernández, 2011

FUENTE ELABORACIÓN PROPIA, CON BASE EN CASADO (20I2), SUÁREZ Y DELGADO (2009), GRAIZBord Y ACUÑA (2005) Y AGUILAR (20II) 
Dos son los elementos distintivos del policentrismo que se da en la ZMVM, según diferentes fuentes. El primero es la importancia que han tenido las cabeceras municipales históricas como embrión de algunos de los actuales subcentros de origen christalleriano (Nava, 2010) (Gráfico 4). El segundo es el papel de las infraestructuras viarias principales en el destino del empleo descentralizado, ya sea orientando la localización de los subcentros (Aguilar \& Alvarado, 2005), o bien conformando auténticos corredores de actividad donde la densidad es prácticamente homogénea a lo largo del eje (Pradilla, 2005).

El patrón de movilidad de la ZMvM captura algunos hechos estilizados de su particular modelo de policentrismo. Durante los últimos veinte años se ha dado una convergencia en el valor del job-ratio por coronas (Casado, 2012), en parte gracias a la emergencia de subcentros. Por tanto, se cumple la condición necesaria -aunque no suficiente- para que el policentrismo genere un patrón de movilidad más eficiente (menores distancias recorridas) que el monocentrismo. El problema es que el policentrismo solo se habría traducido en distancias más cortas de forma parcial. En el lado positivo cabe destacar que, en aquellas zonas donde se ha generado un subcentro, el job-ratio ha aumentado y los desplazamientos exteriores y la distancia media han caído. En el lado negativo, el aumento de la distancia recorrida en los desplazamientos exteriores y el creciente peso de los desplazamientos exteriores respecto de los interiores han comportado un aumento en la distancia recorrida en promedio para el conjunto de la región (Nava, 2010; Graizbord \& Acuña, 2005).

En cuanto a la posible transitoriedad de la estructura policéntrica del empleo, no se ha hallado ningún estudio donde se mida de forma explícita la evolución del peso del empleo en el CBD y subcentros, el método más aceptado para contrastar la hipótesis de Gordon y Richardson (1996). En el caso del ZMVM, la información sobre el porcentaje de empleo espacialmente desagregado por coronas se ha utilizado prioritariamente para señalar el gran peso que sigue reteniendo el área central, o bien la suma de СвD, subcentros y corredores (Aguilar \& Alvarado, 2005; Suárez \& Delgado, 2009). Otros autores han defendido que el policentrismo no está evolucionando hacia la dispersión, sino hacia la conformación de corredores metropolitanos (Pradilla, 2005). 
GRÁFICO 4 | Evolución de la ZMVM y subcentros de empleo identificados
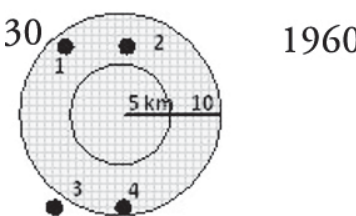

980

995

$: 010$

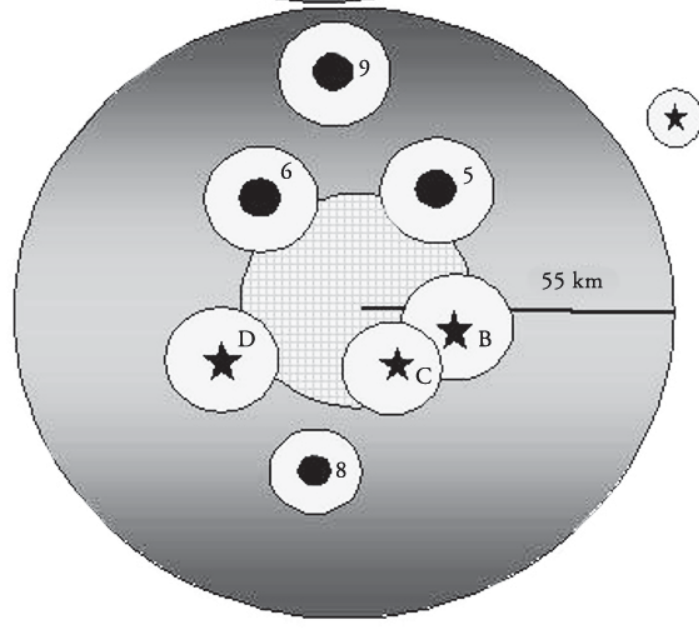

- Centros históricos

1. Azcapotzalco

2. Villa de Gpe.

3. San Ángel

4. Coyoacán

5. Ecatepec

6. Tlalnepantla

7. Naucalpan

8. Tlalpan

Centros históricos

9. Cuautitlán

10. Xochimilco

11. Chalco

12. Texcoco

Subcentros de empleo "históricos"

Æ) Subcentros de empleo "creados"
A. Aeropuerto
B. Pantitlán
C. C. de Abastos

Subcentros de empleo "creados"

D. Santa Fe 


\section{Datos y metodología}

Los datos utilizados en esta investigación son el Censo de Población de 2010 (población, 2010), el conteo de población de 1995 (población, 1995), así como los Censos Económicos de 1994 y 2009 (empleo 1994, empleo 2009). La fuente original de la información, así como de su proyección geográfica, es el Instituto Nacional de Estadística, Geografía e Informática de México (INEGI). El ámbito de observación de los datos es el Área Geoestadística Básica Urbana (AGEB) ${ }^{4}$. Las variables Densidad de población y Densidad de empleo se calculan dividiendo el volumen de población (empleo) por la superficie de cada AGEB o agrupación de varias AGEB. La variable jobratio se calcula dividiendo el empleo por la población ${ }^{5}$. El Índice de Descentralización ${ }^{6}$ aplicado a la población (empleo) es la distancia promedio de cada AGEB respecto del $\mathrm{CBD}$, ponderada por el peso de su población (empleo) respecto del total. El aumento del peso de la población (empleo) de las zonas más periféricas implica un incremento en el valor del índice. El Índice de Concentración aplicado a la población (empleo) es el Índice de Gini ${ }^{7}$.

Para identificar los centros de empleo se ha utilizado una metodología de Umbrales Estadísticos. Consideramos como centro de empleo ( de distintas AGEB con una densidad de empleo superior a la media y un volumen de empleo por encima del 1\% del empleo total. Esta metodología ha sido utilizada en otros trabajos, como García-López y Muñiz (2010). Sus principales virtudes son su simplicidad, su capacidad para adaptarse a ciudades de muy diferente escala, y el hecho de neutralizar el efecto inflacionario que ejerce la fase expansiva del ciclo económico sobre el número de subcentros identificados. Las funciones policéntricas de densidad de población para los años 1995 y 2010 se estiman mediante Mínimos Cuadrados Ordinarios, utilizando como referencia los subcentros identificados en 1994 y 2009, respectivamente. Además de la distancia al CBD y a los subcentros de empleo, se

El inegi define AGEB como "la subdivisión de los municipios que conforman el país (...) su utilidad radica en conformar la unidad básica de muestreo. Tres son sus principales atributos: a) es perfectamente reconocible en el terreno por estar delimitada por rasgos topográficos identificables y perdurables; b) por lo general es homogénea en sus características geográficas, económicas y sociales; c) su extensión es tal que puede ser recorrida a pie. Las AGEB urbanas son aquellas que conforman las localidades con más de 5.000 habitantes".

5 El job-ratio suele calcularse como el ratio entre Puestos de Trabajo Localizados y Población Ocupada Residente. Su uso se circunscribe normalmente a aquellos estudios donde los datos de población y empleo provienen de una misma fuente, lo que garantiza que la suma de empleos y población ocupada coincida a escala agregada. Sin embargo, en nuestro caso, los datos provienen de diferentes fuentes. De hecho, los datos de empleo a nivel agregado son inferiores a los de la población ocupada, debido a que los datos no contienen empleo gubernamental, ni del sector transporte, entre otros. Por esta razón, se ha preferido utilizar como denominador la Población Total (РОв) en lugar de la población ocupada residente (POR).

6 La fórmula empleada: $\mathrm{DCBD}=\frac{1}{\mathrm{~N}} \sum_{\mathrm{i}=1}^{\mathrm{N}} \mathrm{d}_{\mathrm{CBD}_{i}} \frac{\mathrm{B}_{1}}{\mathrm{~B}_{\mathrm{ZMN}}}$, donde $\mathrm{N}$ es el número de unidades de observación, $\mathrm{E}_{\mathrm{i}}$ el empleo en $i, \mathrm{E}_{\mathrm{ZMVM}}$ el empleo en la ZMVM y $\mathrm{d}_{\mathrm{CBDi}}$, la distancia en metros de $i$ al centro.

7 Lee (2007) propone la fórmula, $I G=\sum_{i=1}^{\mathrm{N}} \mathrm{E}_{\mathrm{i}} \mathrm{A}_{\mathrm{i}-1}-\sum_{\mathrm{i}=1}^{\mathrm{N}} \mathrm{E}_{\mathrm{i}-1} \mathrm{~A}_{\mathrm{i}}$, donde $\mathrm{E}_{\mathrm{i}}$ es la proporción acumulada de empleo hasta la unidad de observación $i \mathrm{y}$, paralelamente, $\mathrm{A}_{\mathrm{i}}$, la proporción acumulada de superficie hasta $i$. 
incluye como variable control la altura del AGEB, la distancia a la infraestructura viaria y la distancia a la infraestructura viaria al cuadrado.

Las distancias al CBD, a los subcentros y a la infraestructura viaria, han sido calculadas haciendo uso de un programa SIG. Son, por tanto, distancias euclidianas entre el centroide del polígono formado por cada AGEB y la variable en cuestión; la infraestructura viaria utilizada es la red de carreteras y avenidas más importantes de la ciudad.

\section{La Zona Metropolitana del Valle de México (ZMvM)}

La Zona Metropolitana del Valle de México (zmvm) está ubicada en el suroeste del Valle de México, en la meseta alta del centro del país. Se encuentra a una altitud de más de 2.000 metros, en una cuenca semicerrada. Su superficie es $2.249 \mathrm{~km}^{2}$. Con 19 millones de habitantes, la ZMVM ocupa -junto con Nueva York- el tercer puesto del ranking de ciudades más pobladas del mundo (un Habitat, 2012). La ZMvM está formada por 76 municipios: 16 corresponden al Distrito Federal (DF), 59 al estado de México y uno al estado de Hidalgo.

Durante los quince años que abarca el estudio, la economía mexicana sufrió una serie de shocks con importantes efectos sobre la distribución espacial, tanto de la actividad productiva como de la población. El más significativo es la vertiginosa apertura económica que supuso en 1994 la firma del Tratado de Libre Comercio de América del Norte (TLCAN). En 2010, último año del periodo estudiado, la ZMVM concentraba el 23\% del PIB mexicano y el 18\% de la población (INEGI), dos datos que por sí solos expresan el importante peso que sigue teniendo la ZMVM en el conjunto de la economía del país.

\section{CUADRo 1 | Dinámicas de población}

\begin{tabular}{|c|c|c|c|c|}
\hline & \multicolumn{2}{|c|}{ POBLACIÓN } & \multicolumn{2}{|c|}{ EMPLEO } \\
\hline & 1995 & 2010 & I994 & 2009 \\
\hline Tasa de crecimiento & \multicolumn{2}{|c|}{$21,05 \%$} & \multicolumn{2}{|c|}{$62,25 \%$} \\
\hline Índice de centralización & 14,21 & 15,77 & 9,92 & 10,86 \\
\hline Índice de concentración & 0,54 & 0,461 & 0,692 & 0,633 \\
\hline Ratio de empleo (por cada 100 habitantes) & - & - & 14.92 & 20 \\
\hline
\end{tabular}

FUENTE ELABORACIÓN PROPIA

Durante el periodo estudiado, la población y el empleo aumentaron, siendo el crecimiento del empleo más intenso que el de la población (Cuadro 1). En cuanto a su distribución espacial, al comparar los índices de descentralización y desconcentración (Cuadro 1), aparece que la población se distribuye de forma más descentralizada y desconcentrada que el empleo. No obstante, el empleo se ha descentralizado con más intensidad que la población. El Gráfico 5 muestra la estimación de las tasas de crecimiento del empleo y de la población entre 1994 y 2009 en función de la 
distancia al CBD mediante una función cubic-spline $e^{8}$. Al comparar ambas funciones se observa cómo el patrón descentralizador de la población se reproduce de forma amplificada para el caso del empleo. De aquí se desprende que la relación empleo-población ha tendido a converger a partir de un proceso donde el empleo ha seguido a la población. En conjunto, los datos obtenidos indican una distribución espacialmente más homogénea de la población y del empleo, lo cual, en caso de cumplirse la hipótesis de colocalización, debiera traducirse en una distancia media recorrida más corta en los desplazamientos residencia-trabajo.

GRÁfICO 5| Tasa de crecimiento de población y de empleo y distancia al centro

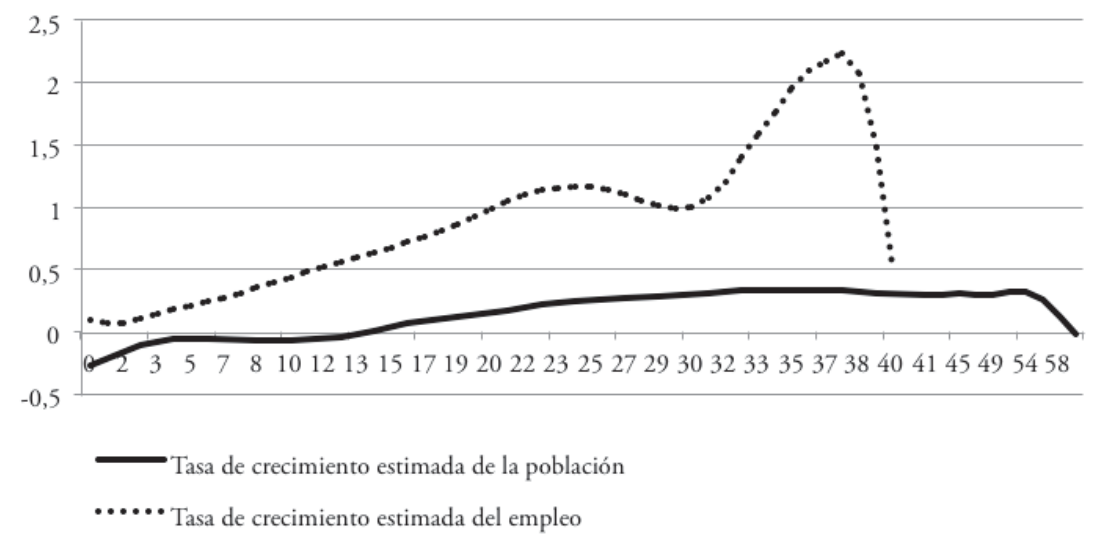

FUENTE ELABORACIÓN PROPIA

GRÁFICo 6| Distribución espacial de población en la ZMVM 2010 (habitantes por ha)

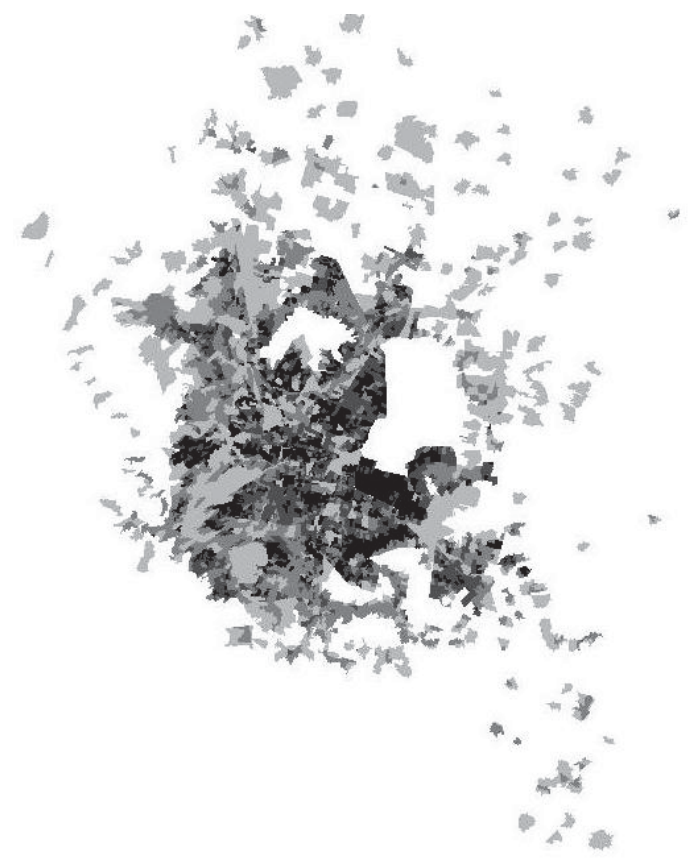

FUENTE ELABORACIÓN PROPIA

Para una revisión exhaustiva de esta función, véase Muñiz et al. (2003). 
El proceso de urbanización de la ZMVM experimentó una aceleración entre los años cuarenta y noventa. El consumo de suelo fue especialmente intenso entre 1970 y 1980. Por otro lado, el crecimiento poblacional de la capital se aceleró entre 1930 y 1970. En 1980, la población de la ZMvM ya superaba los 14 millones. Durante el periodo analizado se observa una cierta ralentización en el consumo de suelo, así como un crecimiento poblacional modesto en comparación con el experimentado en décadas anteriores. Sin embargo, tras esta aparente estabilidad se esconden importantes dinámicas intrametropolitanas. En primer lugar, el proceso descentralizador de la actividad económica ha sido particularmente intenso; en segundo lugar, la población también se ha descentralizado; y en tercer lugar, ha aumentado la integración funcional del sistema metropolitano, lo que se ha traducido en un número creciente de desplazamientos exteriores en automóvil (Garza \& Rodríguez, 1988; Sobrino, 2011).

En relación con la distribución de la población, además de ciertas áreas del centro de la ciudad, existen otras con concentraciones de población particularmente altas: al oriente, Iztapalapa, Ciudad Netzahualcóyotl y Ecatepec; al occidente, Ciudad Satélite, en el municipio de Naucalpan; y el centro de los municipios de Tlalnepantla y Cuautitlán Izcalli. El Gráfico 6 muestra las densidades de población registradas en la ZMVM en 2010. Los grumos de densidad que aparecen coinciden con las áreas antes mencionadas. Todos estos espacios se consolidaron como centros de población de alta densidad entre los años sesenta y ochenta; no obstante, durante las últimas décadas han sido expulsores netos de población (Pradilla, 2005; Nava, 2010).

\section{Resultados}

\section{Identificación de subcentros}

La identificación de subcentros se instrumentó mediante la selección de las áreas de la ciudad que superaban los umbrales estadísticos que se presentan en el Cuadro 2. Ambos umbrales se ajustaron al escenario del empleo del ańo de referencia ${ }^{9}$. Los subcentros identificados en 1994 son siete, a los que hemos denominado según sea su localización. Seis se localizan a menos de $16 \mathrm{~km}$ del свD. Solo Cuautitlán se ubica en la segunda corona (Gráfico 7, Gráfico 8). Por el volumen de empleo que concentran, destacan Tlalnepantla y Central de Abastos, en este orden. En el resto de subcentros, el volumen de empleo oscila entre 30.000 y 35.000. Las localizaciones Central de Abastos (1982) y Aeropuerto emergieron en los últimos cincuenta años. Los restantes subcentros son municipalidades que históricamente han sido polos concentradores de actividad económica y de población. Al aplicar la misma metodología a los datos de empleo de 2009, desaparece el subcentro Aeropuerto, porque no alcanza a satisfacer el umbral de volumen de empleo, y aparece Santa Fe. Santa Fe se localiza en el suroccidente de la ciudad y constituye un importante elemento de equilibrio espacial.

9

Debido a que hemos considerado la misma base geográfica, tanto la densidad de empleo promedio como el volumen de empleo aumentaron en la misma proporción y por encima de la media nacional. 
CUADRo 2 | Umbrales para la identificación de centros de empleo y evolución del empleo dentro y fuera de los centros de empleo

\begin{tabular}{|l|c|c|c|c|c|}
\hline \multirow{2}{*}{ EMPLEO } & \multicolumn{2}{c|}{ I994 } & \multicolumn{2}{c|}{ 2009 } & $\begin{array}{c}\text { TASA DE } \\
\text { CRECIMIENTO }\end{array}$ \\
\hline Densidad mínima & \multicolumn{2}{|c|}{30,2} & \multicolumn{2}{c|}{49,1} & $62,50 \%$ \\
\hline $1 \%$ del empleo & \multicolumn{2}{|c|}{23,494} & \multicolumn{2}{c|}{38,121} & $62,25 \%$ \\
\hline TOTAL & 2.349 .478 & $100 \%$ & 3.812 .144 & $100 \%$ & $62,25 \%$ \\
\hline Subtotal & 1.462 .402 & $62,24 \%$ & 2.105 .027 & $55,22 \%$ & $43,94 \%$ \\
\hline CBD & 1.149 .923 & $48,94 \%$ & 1.576 .127 & $41,34 \%$ & $37,06 \%$ \\
\hline Subcentros & 312.479 & $13,30 \%$ & 528.900 & $13,87 \%$ & $69,26 \%$ \\
\hline Resto & 887.076 & $37,57 \%$ & 1.707 .117 & $45,21 \%$ & $92,44 \%$ \\
\hline
\end{tabular}

FUENTE ELABORACIÓN PROPIA

Estos resultados se hallan en la línea de los obtenidos en Suárez y Delgado (2009) y Casado (2012). Si bien no se identifican centros que no hubiesen sido detectados por Suárez y Delgado, algunos de sus centros no cumplen con nuestro umbral de empleo. Este es el caso de Xochimilco, Chalco, Texcoco, Atizapán y Tepotzotlán (Gráfico 3).

GRÁfICo 7| Subcentros identificados y densidad de población (3D)

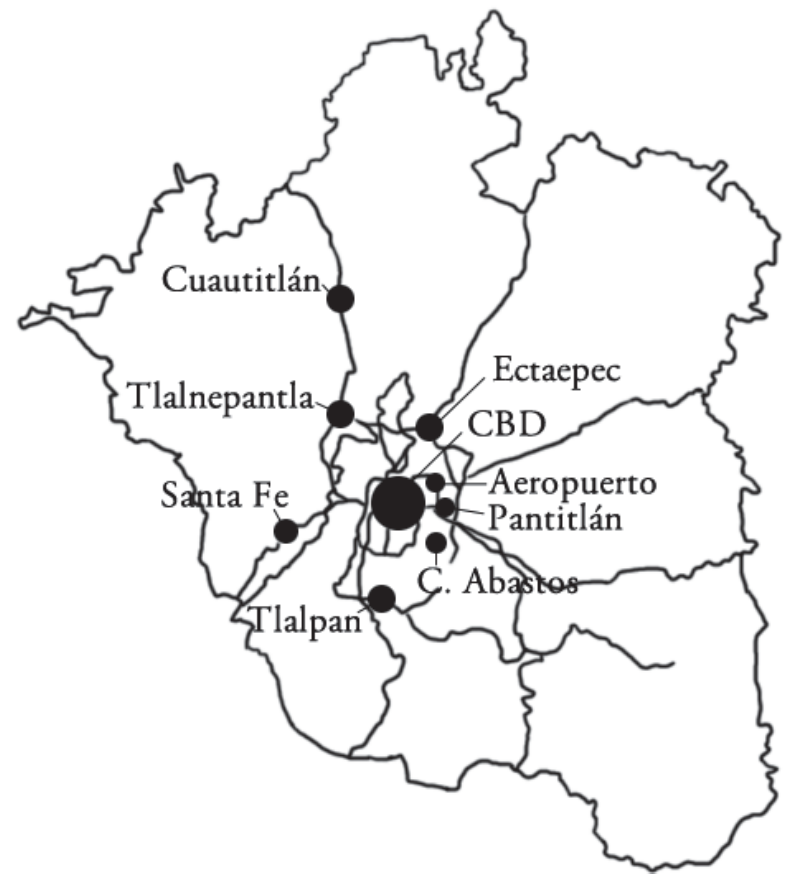

FUENTE ELABORACIÓN PROPIA

El Gráfico 8 muestra una regresión 3D localmente ponderada (LWR) de la densidad de población. En la base hay una silueta de la ciudad con los puntos de la estructura identificada: CBD y subcentros de empleo. La superficie 3D muestra una estimación de la densidad de población en cada AGEB. Cada altura del gráfico se aproximó 
mediante métodos no paramétricos con la observación de densidad de población en cada punto y la de sus más cercanos, sin establecer ninguna relación de causalidad con la estructura urbana de la base. El gráfico es, por tanto, una representación visual del comportamiento de la densidad de población: la existencia de picos de densidad de población en distintos puntos de la ciudad reforzaría la intuición de la existencia de otros elementos estructurantes, más allá del CBD.

GRÁFICo 8| Densidad de población (3D) y subcentros identificados en la ZMVM (2010)

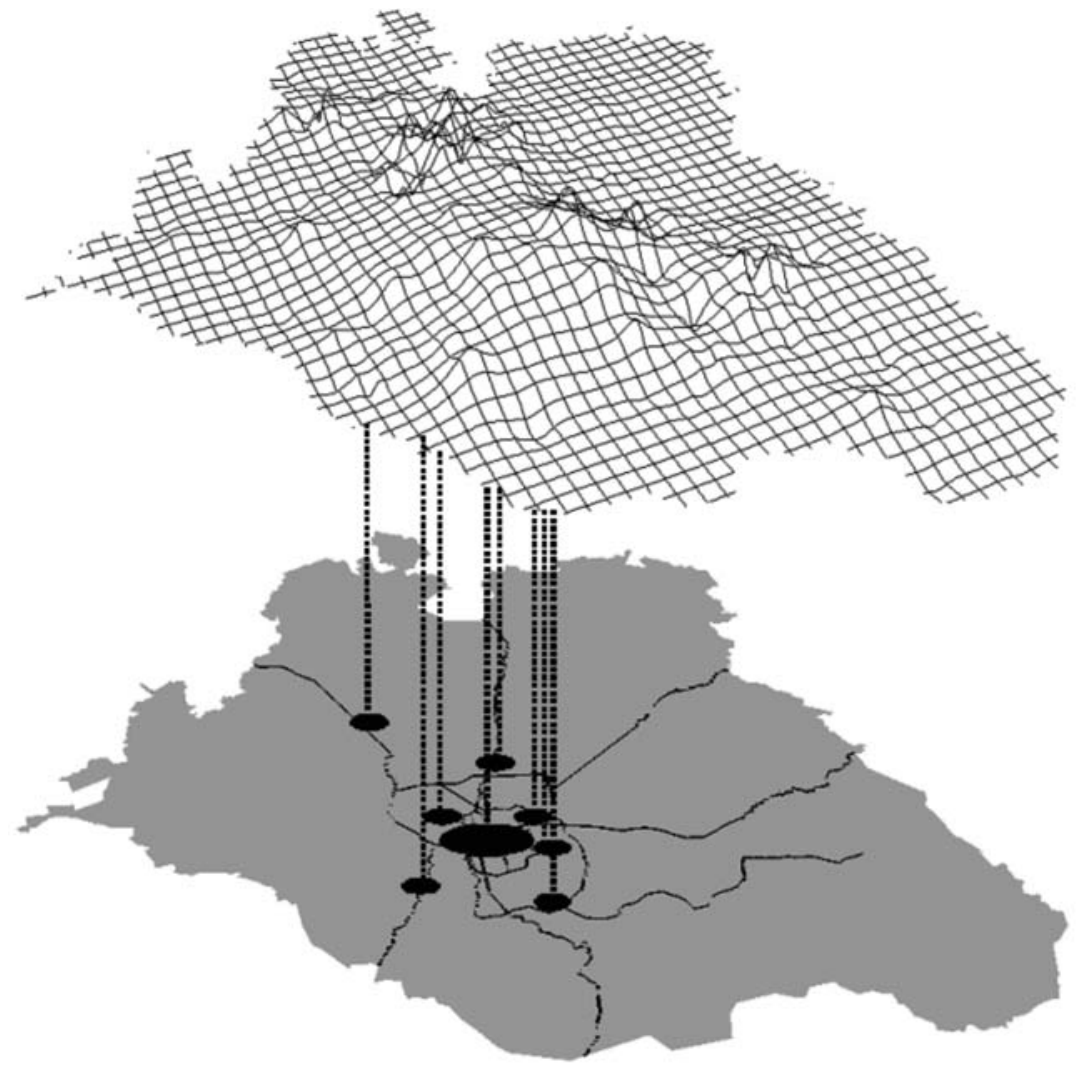

FUENTE ELABORACIÓN PROPIA

Dinámica del empleo 1994-2009: ¿policentrismo o dispersión?

El Cuadro 2 muestra el peso del empleo concentrado en el CBD y subcentros respecto del total, así como su evolución entre 1994 y 2009. En 1994, el porcentaje del empleo localizado en el СвD y subcentros era del 62,3\%. Quince ańos más tarde, dicho porcentaje cae hasta 54,7\%. Estos datos reflejan dinámicas similares a las observadas en otras ciudades de Eeuu y Europa (Lee, 2007; Gilli, 2009; GarcíaLópez \& Muñiz, 2010, Pfister et al. 2000). Es importante seńalar que durante dicho periodo, la pérdida de peso del СвD no se ha traducido en un incremento del peso de los subcentros. De hecho, el dinamismo en términos de empleo del CBD es superior al de los subcentros. Tomados en conjunto, estos resultados apuntan hacia la posible transitoriedad del modelo policéntrico, que evolucionaría hacia un modelo más disperso, donde el CBD seguiría manteniendo un peso relevante. 
Estimación de la función policéntrica de densidad de población

Los principales resultados que se desprenden del análisis de regresión donde, además de la distancia al СвD y de las variables de control, se incluye como regresor la distancia al subcentro más cercano de los años 1994 y 2009, son los siguientes: a) la capacidad explicativa global de la función policéntrica de densidad $\left(\mathrm{R}^{2}\right)$ prácticamente no supera la de la función monocéntrica, lo cual indica que la ZMvM presenta una fuerte inercia monocéntrica; b) el gradiente del subcentro más próximo resulta significativo y con el signo adecuado (columnas 3 y 5 del Cuadro 3). Este resultado permite matizar la afirmación anterior, ya que supone evidencia empírica favorable de un policentrismo estructurante, es decir, capaz de ajustar la densidad de población; c) la caída en la significatividad del gradiente del subcentro más próximo indicaría que la población tendría cada vez menos en cuenta la proximidad a un subcentro a la hora de asignar valor a una determinada localización. Esto implicaría que las ventajas que, en términos de movilidad, ofrecería el policentrismo respecto del monocentrismo, no llegarían a alcanzarse; d) la topografía o rugosidad del terreno importa. La variable control altitud presenta un signo negativo y resulta estadísticamente significativa, recogiendo la dificultad que supone construir en altura cuando la pendiente es elevada; y e) la proximidad a la infraestructura viaria principal también afecta el valor de la densidad. En el caso de la infraestructura viaria, los resultados provisionales ofrecían un signo (positivo) contrario al esperado (negativo). Que la densidad aumente con la distancia a la infraestructura viaria resulta difícilmente explicable, ya que el suelo más próximo a la infraestructura viaria debería valorarse más (Steen, 1986; García-López, 2012). Sin embargo, al añadir como regresor la variable al cuadrado, el coeficiente obtenido resulta estadísticamente significativo y con un signo negativo, lo que indica que existe un fenómeno de expulsión que afecta un espacio de unos $6,5 \mathrm{~km}$ alrededor de los ejes de transporte. A partir de esta distancia, la densidad se comportaría conforme a lo esperado, es decir, cae al aumentar la distancia. A diferencia de lo que sucede con el gradiente del subcentro más próximo, los parámetros asociados a la distancia a la infraestructura de transporte resultan cada vez más significativos y su valor aumenta con el paso del tiempo. Esto parecería avalar la idea de que la ZMVM está evolucionando hacia un modelo espacial más tentacular que policéntrico (Cuadro 3), en el que la infraestructura viaria estaría desempeñando un papel determinante en la organización de las funciones del espacio.

Las estimaciones que aparecen en el Cuadro 3 para los ańos 1995 y 2010 validan la capacidad estructurante de los subcentros, ya que demuestran afectar las condiciones de densidad conforme a lo esperado. Aun así, hay que tener en cuenta que el modelo estimado impide conocer el comportamiento de cada subcentro de forma individual. A continuación se muestran los resultados obtenidos al estimar un modelo completo, donde se toman como variables explicativas las distancias a todos los subcentros identificados (Cuadro 4). Para corregir los problemas de multicolinealidad, en lugar de la distancia directa a los subcentros se toma la inversa de la distancia, restringiendo con ello su radio de acción. Como resultado, la lectura de los coeficientes es la inversa a lo habitual; es decir, un signo positivo indica que la densidad decrece con la distancia. 
CUADro 3 | Resultados de la estimación de parámetros de funciones monocéntrica y policéntrica con el subcentro más cercano por oLS

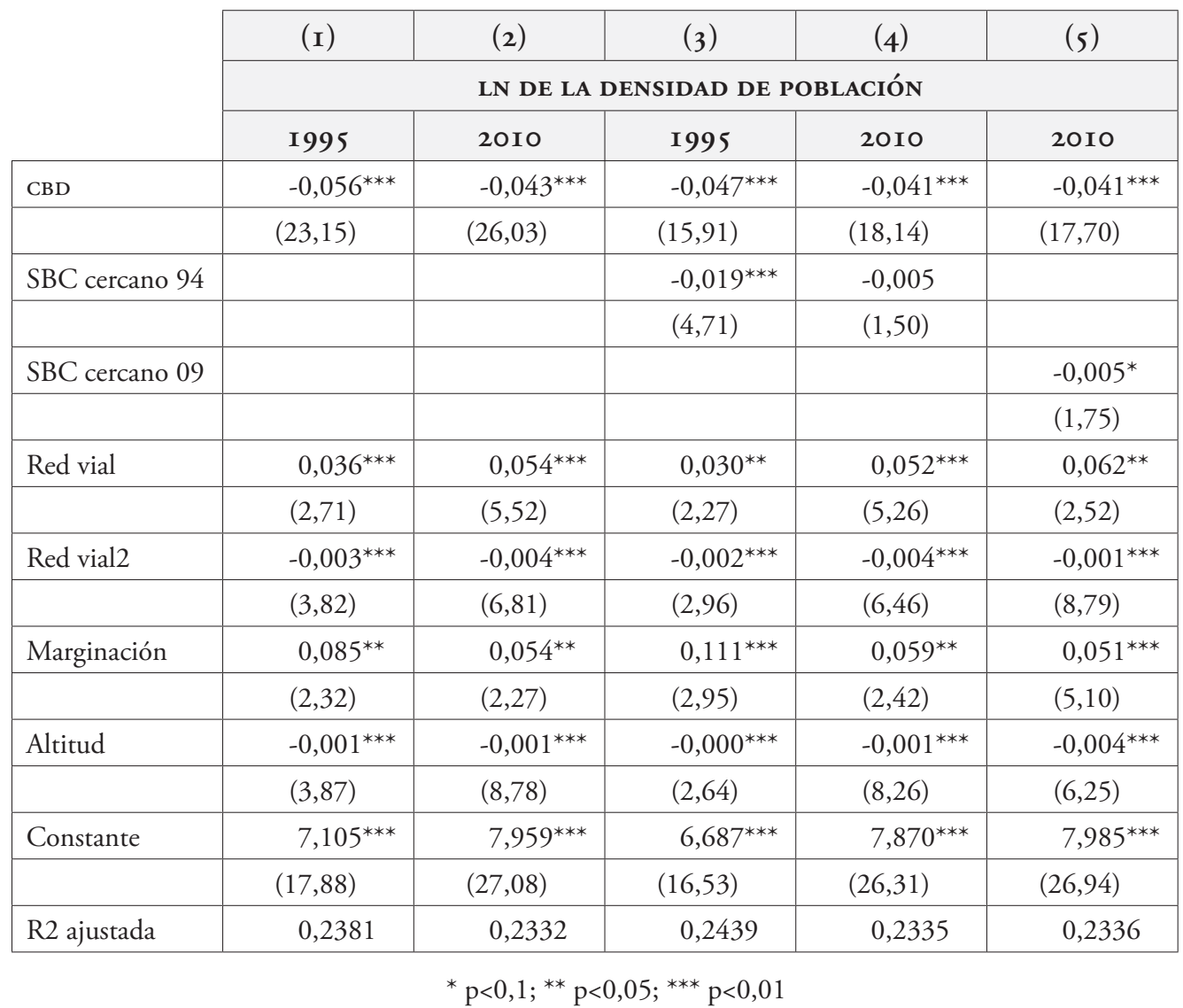

FUENTE ELABORACIÓN PROPIA

Hay dos resultados que merecen atención. De un lado, el signo del gradiente es, en general, el correcto para los subcentros tanto para los surgidos de la descentralización reciente del empleo como para los históricos; este aportaría evidencia a favor de la capacidad estructurante de los subcentros en el sentido de que predice la teoría; no obstante, esto no es así con los asociados a los subcentros: Aeropuerto, Santa Fe y Tlalnepantla, todos ellos estadísticamente significativos (Cuadro 4, columnas 1, 2 y 3). El primero puede explicarse por las externalidades negativas de un aeropuerto sobre población que vive cerca; el segundo, por el proceso de formación de un subcentro de empleo; el tercero, por presentar un patrón tradicionalmente caracterizado por una baja densidad de población y, al mismo tiempo, por la proximidad a áreas de alta densidad (Ciudad Satélite y Cuautitlán Izcalli).

Por otro lado, la capacidad explicativa del modelo es muy limitada y con tendencia a la baja, en línea con el análisis anterior. Una posible explicación de este resultado es la presencia de grumos de densidad de población en áreas que no cuentan con concentraciones de empleo suficientes para constituir un subcentro de empleo. Es decir, un componente de segregación del suelo residencial respecto del de carácter productivo, componente reforzado por las políticas de urbanización que 
premiaron la construcción de unas periferias densas y compactas, así como por la expulsión de población en el centro de población, espoleada por el sismo de 1985.

La hipótesis de colocalización de la población y el empleo implica que, a menos de estar saturado, el suelo más próximo a los centros de empleo debería urbanizarse con unos niveles de densidad decrecientes al aumentar la distancia. Las regresiones policéntricas inversas de carácter dinámico, donde la variación en la densidad de población se explica en función de los mismos regresores que aparecen en la especificación estática ${ }^{10}$ (Cuadro 4, columnas 4 y 5), complementan la valoración del efecto de la estructura sobre la intensidad del uso del suelo destinado a la residencia mediante la contrastación del impacto de la estructura sobre los cambios en la distribución de la población. De acuerdo con el resultado de este último ejercicio, la estructura es una fuente de organización de la dinámica poblacional en la ZMVM: los parámetros estimados son estadísticamente significativos. Sin embargo, su efecto apunta en sentido contrario al de la hipótesis de colocalización: tanto el CBD como los subcentros en general parecen expulsar a la población ${ }^{11}$, resultado consistente con la existencia de una creciente desconexión entre los mercados de vivienda y de trabajo, tal como apuntaba la caída del valor de la $\mathrm{R}^{2}$ correspondiente a las estimaciones estáticas para los años 1995 y 2010.

CUADRo 4 Resultados de la estimación de parámetros de función policéntrica inversa estática y dinámica con todos los subcentros de empleo por oLs

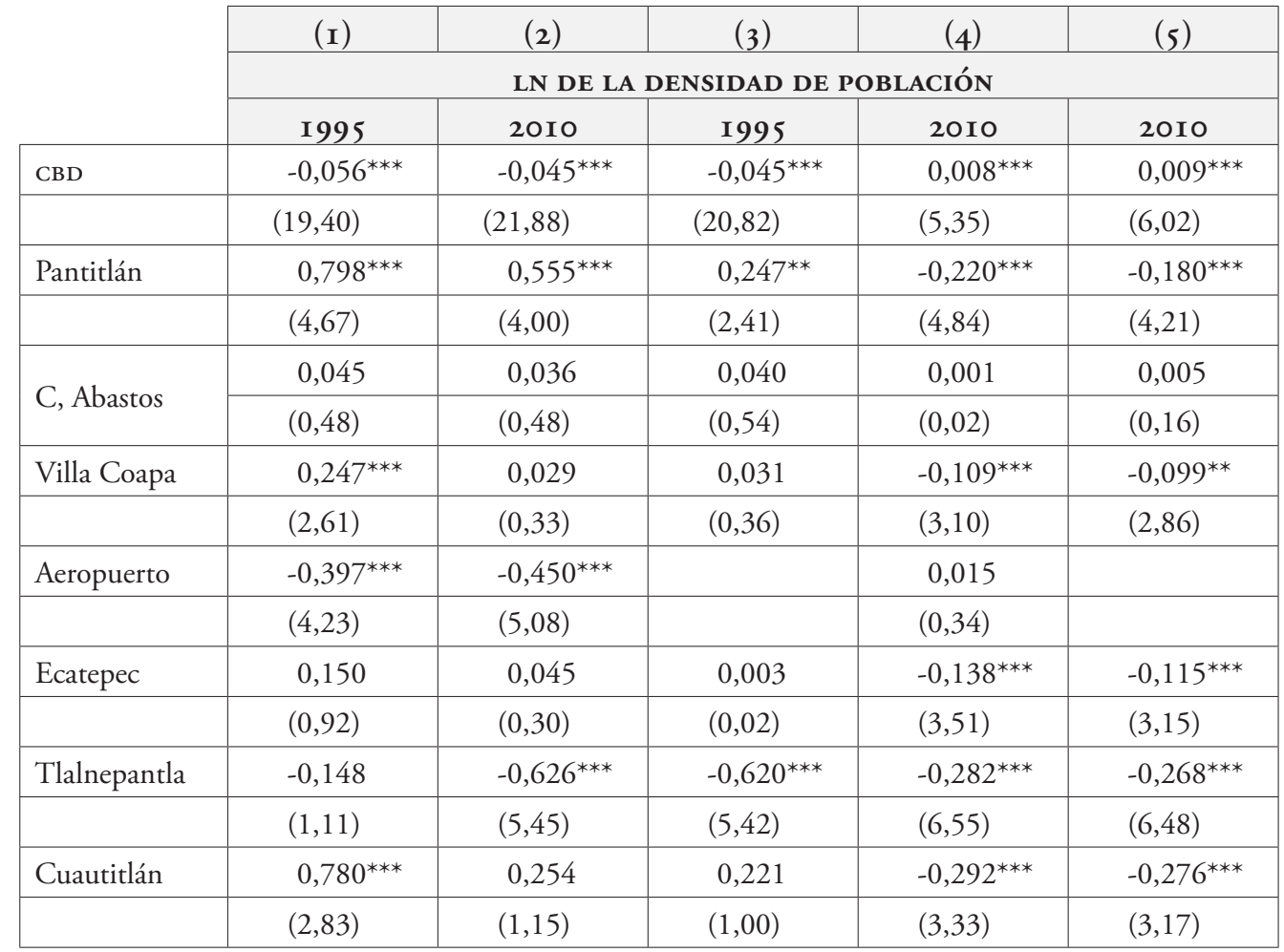

10 Se estiman los parámetros de la función $\ln D_{i, t}-\ln D_{i, t-1}=y_{1}+y_{2} D I S T_{C B D i}+\sum_{n=1}^{N} \beta_{n} D I S T_{n_{i}}+e_{i}$

11 Solo Santa Fe parece atraer población, lo que corroboraría la explicación de que este subcentro se halla en proceso de consolidación. 
(continuación)

\begin{tabular}{|c|c|c|c|c|c|}
\hline & & & & & 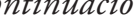 \\
\hline & (I) & $(2)$ & (3) & $(4)$ & $(5)$ \\
\hline & \multicolumn{5}{|c|}{ LN DE LA DENSIDAD DE POBLACIÓN } \\
\hline & I995 & 2010 & I995 & 2010 & 2010 \\
\hline \multirow[t]{2}{*}{ Santa Fe } & & & $-0,539^{*}$ & & $0,293^{* * *}$ \\
\hline & & & $(1,65)$ & & $(3,33)$ \\
\hline \multirow[t]{2}{*}{ Red Vial } & $0,040^{* * *}$ & $0,055^{* * *}$ & $0,056^{* * *}$ & $0,025^{* * *}$ & $0,026^{* * *}$ \\
\hline & $(2,97)$ & $(5,44)$ & $(5,55)$ & $(4,05)$ & $(4,19)$ \\
\hline \multirow[t]{2}{*}{ Red vial2 } & $-0,003^{* * *}$ & $-0,004^{* * *}$ & $-0,004^{* * *}$ & $-0,001^{* * *}$ & $-0,001^{* * *}$ \\
\hline & $(3,76)$ & $(6,45)$ & $(6,61)$ & $(4,19)$ & $(4,33)$ \\
\hline \multirow[t]{2}{*}{ Marginación } & $0,101^{* *}$ & $0,048^{*}$ & 0,037 & $0,096^{* * *}$ & $0,101^{* * *}$ \\
\hline & $(2,53)$ & $(1,89)$ & $(1,44)$ & $(5,05)$ & $(5,35)$ \\
\hline \multirow[t]{2}{*}{ Altitud } & $-0,001^{* * *}$ & $-0,001^{* * *}$ & $-0,001^{* * *}$ & $-0,000$ & $-0,000$ \\
\hline & $(3,38)$ & $(8,64)$ & $(7,22)$ & $(0,45)$ & $(1,18)$ \\
\hline \multirow[t]{2}{*}{ Constant } & $6,853^{* * *}$ & $8,002^{* * *}$ & $7,762^{* * *}$ & 0,112 & 0,222 \\
\hline & $(16,24)$ & $(25,85)$ & $(24,31)$ & $(0,51)$ & $(1,02)$ \\
\hline R2 ajustada & 0,2448 & 0,2405 & 0,2388 & 0,1284 & 0,1308 \\
\hline
\end{tabular}

FUENTE ELABORACIÓN PROPIA

La creciente desconexión entre las áreas residenciales y los centros de empleo indica que el policentrismo de la ZMVM difícilmente corregirá los problemas de movilidad de la región; es decir, frente el monocentrismo tradicional, el policentrismo emergente no parece capacitado para reducir la distancia de los desplazamientos o aumentar el número de desplazamientos a pie o en transporte público. Por lo tanto, reducir el impacto de la movilidad requerirá un sistema de transporte público jerarquizado, extenso y capacitado para soportar un volumen creciente de desplazamientos externos, que en la actualidad solo pueden realizarse en auto privado.

\section{Resumen y conclusiones}

Los principales resultados de esta investigación pueden resumirse en los siguientes puntos:

1. El empleo de la ZMVM está más centralizado y más concentrado que la población.

2. Durante el periodo estudiado, la descentralización del empleo ha sido más intensa que la de la población.

3. Se han identificado siete subcentros de empleo.

4. El porcentaje de puestos de trabajo en el CBD y subcentros ha caído con el paso del tiempo, lo cual parece indicar una tendencia hacia la dispersión del empleo que podría a su vez impulsar la dispersión de la población.

5. Los gradientes de los subcentros presentan en general el signo adecuado, es decir, la densidad de población cae con la distancia al subcentro. 
6. Sin embargo, la capacidad explicativa global de la función de densidad policéntrica no es sensiblemente superior a la de la monocéntrica.

7. Esta limitación en la capacidad explicativa del modelo se debe a dos factores: a) La aparente desconexión entre los espacios con altas densidades de empleo, por un lado, y de población, por otro; es lo ocurrido en municipios como Netzahualcóyotl, Valle de Chalco, Iztapalapa o Ciudad Satélite, en los que se promovieron densos asentamientos mediante políticas gubernamentales o por medio del mercado. Y b) la disminución de la población en el centro de la ciudad espoleada por el sismo de 1985.

8. La caída en la significatividad del gradiente de los subcentros indica una creciente desconexión entre los mercados de vivienda y de trabajo.

9. Los resultados de la estimación de la función dinámica muestran que la variación en la distribución de la población está afectada por la localización de los centros de empleo, pero en el sentido contrario al esperado. Es decir, la población ha tendido a concentrarse a una cierta distancia de los subcentros de empleo.

10. Los coeficientes de las variables de infraestructura viaria son cada vez más significativos, lo que avalaría la idea de que la estructura espacial de la ZMvM estaría evolucionando desde el policentrismo hacia una estructura tentacular, donde el empleo descentralizado se localiza preferentemente en corredores de actividad.

11. En términos de política, para frenar los crecientes costes de congestión, debería desarrollarse infraestructuras viales y sistemas de transporte público dimensionados a escala metropolitana, es decir, capaces de absorber un volumen creciente de desplazamientos externos.

\section{Referencias bibliográficas}

Abramo, P. (2011). La ciudad com-fusa: mercado y producción de la estructura urbana en las grandes metrópolis latinoamericanas. EURE, 38(114), 35-69. http://dx.doi. org/10.4067/S0250-71612012000200002

Aguilar, A. G. (2002). Las megaciudades y las periferias expandidas. Ampliando el concepto en ciudad de México. EURE, 28(85), 121-149. En http://www.eure.cl/wp-content/ uploads/2002/12/EURE_85_06_AGUILAR.pdf

Aguilar, A. G. \& Alvarado, C. (2005). La reestructuración del espacio urbano de la Ciudad de México. ¿¿Hacia la metrópoli multinodal? En A. G. Aguilar (Ed.), Procesos metropolitanos grandes ciudades. Dinámicas recientes en México y otros paises (pp. 265-308). México: Porrúa.

Aguilar, A. G. \& Hernández, J. (2011). Metropolitan transformation and polycentric structure in Mexico City. Identification of urban subcenters 1989-2009. Presentado en IGU Urban Commission Meeting 2011, Canterbury Christ Church University, Canterbury, UK, agosto 14-20, 2011. En http://www.unil.ch/webdav/site/igu-urban/shared/ Aguilar_Hernandez.ppt 
Aguilera, A. (2005). Growth in commuting distances in French polycentric metropolitan areas: Paris, Lyon and Marseille. Urban Studies, 42(9), 1537-1547. doi: 10.1080/00420980500185389

Ahlfeldt, G. \& Wendland, N. (2013). How polycentric is a monocentric city: Centres, spillovers and hysteresis. Journal of Economic Geography, 13(1), 53-83. doi: 10.1093/jeg/lbs013

Alonso, W. (1960). A theory of the urban land market. Regional Science Association, Papers and Proceedings, 6(1), 149-157. doi: 10.1111/j.1435-5597.1960.tb01710.x

Alperovich, G. \& Deutsch, J. (1994). Joint estimation of population density functions and the location of the Central Business District. Journal of Urban Economics, 36(3), 239-248. doi: $10.1006 /$ juec. 1994.1034

Arellano, B. E. \& Roca, J. (2010). El urban sprawl ¿̨un fenómeno de alcance planetario? Los ejemplos de México y España. ACE, Architecture, City and Environment, 4(12), 115-148. Separata electrónica en http://upcommons.upc.edu/revistes/bitstream/2099/8556/7/ ACE_12_ST_30.pdf

Avendaño, A. (2013). Polycentrism in Bogotá: Substitution or complementary relationships of subcenters with CBD. Tesis Doctoral, Departament Economia Aplicada, Universitat Autònoma de Barcelona.

Becerril-Padua, M. (2000). Policentrismo en las ciudades latinoamericanas. El caso de Santiago de Chile. Theomai, 1. En http://www.redalyc.org/articulo.oa?id=12400108

Bertaud, A. (2004). The spatial organization of cities: Deliberate outcome or unforeseen consequence? Working paper, University of California, Institute of Urban and Regional Development, 01. En http://hdl.handle.net/10419/23612

Boderman, J. E. (1998). The suburbanization of the institutional investment advisory industry. Metropolitan Philadelphia 1983-1993. Professional Geographer, 50(1), 112-126. doi: $10.1111 / 0033-0124.00108$

Borsdorf, A. (2003). Cómo modelar el desarrollo y la dinámica de la ciudad latinoamericana. EURE, 29(86), 37-49. http://dx.doi.org/10.4067/S0250-71612003008600002

Casado, J. M. (2012). La estructura policéntrica de los mercados laborales locales de la Zona Metropolitana del Valle de México. Investigaciones Geográficas [Boletín Instituto de Geografía, UNAM], 79, 97-118. doi: http://dx.doi.org/10.14350/rig.34540

Craig, S. \& Kohlhase, J. (2010). Employment subcenters and the distribution of economic activity. University of Houston working paper. Borrador preliminar en http://www. uh.edu/ -kohlhase/Craig_Kohlhase_subcenters_WP_5_2010.pdf

Cervero, R. \& Wu, K-L. (1998). Polycentrism, commuting, and residential location in the San Francisco Bay area. Environment and Planning A, 29, 865-886.

Champion, A. G. (2001). A changing demographic regime and evolving polycentric urban regions: Consequences for the size, composition and distribution of city populations. Urban Studies, 38, 657-677. doi: 10.1080/00420980120035277

De Mattos, C. A. (1999). Santiago de Chile, globalización y expansión metropolitana: lo que existía sigue existiendo. EURE, 25(76). http://dx.doi.org/10.4067/S025071611999007600002

Dowall, D. E. \& Treffeisen, A. (1991). Spatial transformation in cities of the developing world. Multinucleation and land-capital suburbanisation in Bogotá, Colombia. Regional Science and Urban Economics, 21(2), 201-224. doi: 10.1016/0166-0462(91)90034-K 
Gallo, M. T., Garrido, R. \& Vivar, M. (2010). Cambios territoriales en la Comunidad de Madrid: policentrismo y dispersión. EURE, 36(107), 5-26. En http://www.eure.cl/ wp-content/uploads/2010/04/EURE_107_01_GALLO.pdf

García-López, M. A. (2006). Estructura espacial del empleo y economías de aglomeración: el caso de la industria en la RMB. Tesis Doctoral. DEA UAB [Mimeo].

García-López, M. A. (2012). Urban spatial structure, suburbanization and transportation in Barcelona. Journal of Urban Economics, 72(2-3), 176-190. doi: 10.1016/j. jue.2012.05.003

García-López, M. A. \& Muñiz, I. (2010). Employment decentralisation: Polycentricity or scatteration? The case of Barcelona. Urban Studies, 47, 3035-3056. doi: $10.1177 / 0042098009360229$

Garza, G. \& Rodríguez, F. (Comps.). (1988). Normatividad urbanistica en las principales metrópolis de México. México, D.F.: El Colegio de México.

Gilli, F. (2009). Sprawl or reagglomeration? The dynamics of employment deconcentration and industrial transformation in Greater Paris. Urban Studies, 46, 1385-1420. doi: $10.1177 / 0042098009104571$

Giuliano, G. (1995). The weakening transportation-land use connection. Access, 6, 3-11.

Giuliano, G. \& Redfearn, C. (2005). Not all sprawl: Evolution of employment concentrations in Los Angeles, 1980-2000. Submitted to Urban Studies for publication consideration. En http://lusk.usc.edu/sites/default/files/working_papers/wp_2005-1002.pdf

Giuliano, G. \& Small, K. A. (1991). Subcenters in the Los Angeles Region. Regional Science and Urban Economics, 21(2), 163-182. doi: 10.1016/0166-0462(91)90032-I

Glaeser, E. L. \& Gottlieb, D.(2009). The wealth of cities: Agglomeration economies and spatial equilibrium in the United States. Journal of Economic Literature, 47, 983-1028. doi: $10.3386 / \mathrm{w} 14806$

Gonzales de Olarte, E. \& Del Pozo Segura, J. M. (2012). Lima, una ciudad policéntrica. Un análisis a partir de la localización del empleo. Investigaciones Regionales, 23, 29-52. En http://www.aecr.org/images/ImatgesArticles/2012/10/Gonzales.pdf

Gordon, P. \& Richardson, H. W. (1996). Beyond polycentricity. The dispersed metropolis, Los Angeles, 1970-1990. Journal of the American Planning Association, 62(3), 289-295. doi: 10.1080/01944369608975695

Gordon, P., Richardson, H. W. \& Wong, L. (1986). The distribution of population and employment in a polycentric city - The case of Los-Angeles. Environment and Planning A, 18(2), 161-173. doi: 10.1068/a180161

Graizbord, B. \& Acuña, B. (2005). La estructura polinuclear del Área Metropolitana. En A. G. Aguilar (Coord.), Procesos metropolitanos y grandes ciudades. Dinámicas recientes en México y otros países (pp. 309-328). México, D.F.: Porrúa.

Handy, S. (1996). Methodologies for exploring the link between urban form and travel behavior. Transportation Research D, 1(2), 151-165. Doi: 10.1016/S1361-9209(96)00010-7

Heinrichs, D., Nuissl, H. \& Rodríguez, C. (2008). Dispersión urbana y nuevos desafíos para la gobernanza (metropolitana) en América Latina: el caso de Santiago de Chile. EURE, 35(104), 29-46. http://dx.doi.org/10.4067/S0250-71612009000100002

Janoschka, M. (2002). El nuevo modelo de la ciudad latinoamericana: fragmentación y privatización. EURE, 27(85), 11-29. http://dx.doi.org/10.4067/S025071612002008500002 
Lee, B. (2007). "Edge" or "edgeless" cities? Urban spatial structure in U.S. Metropolitan Areas 1980 to 2000. Journal of Regional Science, 47, 479-515. doi: 10.1111/j.14679787.2007.00517.x

Ludueña, W. (2006). Ciudad y patrones de asentamiento. Estructura urbana y tipologización para el caso de Lima. EURE, 32(95), 37-59. http://dx.doi.org/10.4067/S025071612006000100003

Lungo, M. (2000). Urban sprawl and land regulation in Latin America. Land Lines, 13(2). En http://www.lincolninst.edu/pubs/255_Urban-Sprawl-and-Land-Regulation-inLatin-America

McMillen, D. P. \& McDonald, J. F. (1998). Population density in suburban Chicago: A bidrent approach. Urban Studies, 35, 1119-1130. doi: 10.1080/0042098984510

McMillen, D. P. \& Lester, T. W. (2003). Evolving subcenters: Employment and population densities in Chicago, 1970-2020. Journal of Housing Economics, 12(1), 60-81. doi: 10.1016/S1051-1377(03)00005-6

Meijers, E. J. \& Burger, B. J. (2010). Spatial structure and productivity in US metropolitan areas. Environment and Planning A, 42, I383-1402. doi: 10.1068/a42151

Mills, E. S. (1967). An aggregative model of resource allocation in a metropolitan area. The American Economic Review, 57(2), 197-210. En http://www.vwl.tuwien.ac.at/hanappi/ AgeSo/rp/Mills_1967.pdf

Monkkonen, P. (2011). Do Mexican cities sprawl? Housing-finance reform and changing patterns of urban growth. Urban Geography, 32, 406-423. doi: 10.2747/02723638.32.3.406

Muñiz, I. \& García-López, M. A. (2013). Anatomía de la dispersión urbana en Barcelona. EURE, 39(116), 189-219. http://dx.doi.org/10.4067/S0250-71612013000100008

Muñiz, I., Galindo, A. \& García-López, M. A. (2003). Cubic spline density functions and satellite city delimitation: the case of Barcelona. Urban Studies, 40, 1303-1321. doi: 10.1080/0042098032000084613

Muñiz, I. \& García-López, M. A. (2012). Chaos and order in the contemporary city. The impact of urban spatial structure on population density and commuting distance in Barcelona, 1986-2001. Working Paper del Departamento de Economía Aplicada de la Universitat Autònoma de Barcelona (UAB). En http://ddd.uab.es/record/102152?ln=es

Muñiz, I. \& García-López, M. A. (2009). Policentrismo y sectores intensivos en información y conocimiento. Ciudad y Territorio. Estudios Territoriales, 160, 263-289.

Muñiz, I., García-López, M. A. \& Galindo, A. (2008). The effect of employment subcentres on population density in Barcelona. Urban Studies, 45, 627-649. doi: $10.1177 / 0042098007087338$

Muth, R. M. (1961). The spatial structure of the housing market. Papers in Regional Science, 7(1), 207-220. doi: 10.1111/j.1435-5597.1961.tb01780.x

Nava, E. (2010). Policentralidad y movilidad en el Área Metropolitana de la Ciudad de México: variables relacionadas con la capacidad de atracción de viajes. Presentado en la $X$ Reunión Nacional de Investigación Demográfica, Sociedad Mexicana de Demografía, El Colegio de México-Inegi-IIs unam-El Colegio de la Frontera Norte-Sep-ConApoUnFPA, México, D.F., del 3 al 6 de noviembre de 2010. 
Ojima, R. \& Hogan, D. (2009). The demographic composition of urban sprawl: Local and regional challenges concerning global environmental change in Brazilian metropolitan areas. Presentado en XVI IUSSP [International Union for the Scientific Study of Population], International Population Conference, Sesión 191, octubre 2, 2009. En http://iussp2009. princeton.edu/papers/90294

Pfister, N., Freestone, R. \& Murphy, P. (2000). Polycentricity or dispersion? Changes in center employment in Metropolitan Sydney. Urban Geography, 21, 428-442. doi: 10.2747/0272-3638.21.5.428

Phelps, N. A. \& Parsons, N. (2003). Edge Urban Geographies: Notes from the margins of Europe's capital cities. Urban Studies, 40, 1725-1749. doi: 10.1080/0042098032000106573

Pradilla, E. (2005). Zona Metropolitana del Valle de México: Megaciudad sin proyecto. Ciudades: Revista del Instituto Universitario de Urbanistica de la Universidad de Valladolid, 9, 83-104. En http://dialnet.unirioja.es/servlet/articulo?codigo=2230701

Roca, J., Marmolejo, C. \& Moix, M. (2009). Urban structure and polycentrism: Towards a redefinition of the sub-centre concept. Urban Studies, 46, 2841-2868. doi: $10.1177 / 0042098009346329$

Rojas, C. A., Muñiz, I. \& García-López, M. A. (2009). Estructura urbana y policentrismo en el Área Metropolitana de Concepción. EURE, 35(105), 47-70. http://dx.doi. org/10.4067/S0250-71612009000200003

Roitman, S. \& Phelps, N. (2010). Do gates negate the city? Gated communities' contribution to the urbanization of suburbia in Pilar, Argentina. Urban Studies, 48, 3487-3509. doi: $10.1177 / 0042098010397395$

Romein, A., Fernández-Maldonado, A. M. \& Verkoren, O. (2009). Polycentric metropolitan form: Application of a "Northern" concept in Latin America. Metropolitan Form, 5 (Autumn), 127-145. En http://dspace.library.uu.nl/handle/1874/289110

Rubiera, F. \& Aponte, E. (2009). Retos para el crecimiento equilibrado de la ciudad Bogotá. El declive del centro y el desamparo de la periferia meridional. Investigaciones Regionales, 16, 43-69. En http://dialnet.unirioja.es/servlet/articulo?codigo=3104997

Sobrino, J. (2011). La urbanización en el México contemporáneo. Presentado en Reunión de Expertos sobre "Población, Territorio y Desarrollo Sostenible", en Santiago de Chile del 16 al 17 de agosto del 2011. Santiago: CEPAL. En http://www.eclac.cl/celade/noticias/ paginas/5/44305/Jaime_Sobrino.pdf

Steen, R. C. (1986). Non ubiquitous transportation and urban population density gradients. Journal of Urban Economics, 20, 97-106. DOI: 10.1016/0094-1190(86)90017-3

Suárez, M., \& Delgado, J. (2009). Is Mexico City polycentric? A trip attraction capacity approach. Urban Studies, 46, 2187-2211. doi: 10.1177/0042098009339429

Shukla, V. \& Waddell, P. (1991). Firm location and land use in discrete urban space. A study of the spatial structure of Dallas-Fort worth. Regional Science and Urban Economics, 21(2), 225-253. doi: 10.1016/0166-0462(91)90035-L

Sullivan, M. (1986). A general equilibrium model with agglomerative economies and decentralized employment. Journal of Urban Economics, 20(1), 55-74. doi: 10.1016/0094-1190(86)90015-X

Shearmur, R. \& Coffey, W. J. (2002). A tale of four cities: intrametropolitan employment distribution in Toronto, Montreal, Vancouver, and Ottawa-Hull, 1981-1996. Environment and Planning A, 34(4), 575-598. 
un Habitat. (2012). State of the world's cities 2010/2011. Bridging the Urban Divide. En http:// sustainabledevelopment.un.org/content/documents/11143016_alt.pdf

Vecslir, L. \& Ciccolella, P. (2012). Dinámicas, morfologías y singularidades en la reestructuración metropolitana de Buenos Aires. Revista Iberoamericana de Urbanismo, 8, 23-41.

Veneri, P. (2010). The identification of sub-centres in two Italian metropolitan areas: A functional approach. Quaderno di Ricerca, 343 (Dipartimento di Scienze Economiche e Sociali, Università Politecnica Delle Marche/upm). Publicado posteriormente en Cities, 31 (abril 2013), 177-185, doi: 10.1016/j.cities.2012.04.006

Weber, J. (2003). Individual accessibility and distance from major employment centers: An examination using space time measures. Journal of Geographical Systems, 5(1), 51-70. doi: $10.1007 / \mathrm{s} 101090300103$

White, M. J. (1999). Urban areas with decentralized employment: Theory and empirical work. En E. S. Mills \& P. Cheshire (Eds.), Handbook of Regional and Urban Economics (vol. 3, pp. 1375-1412). En http://econweb.ucsd.edu/ - miwhite/handbook-urban-white.pdf

Wieand, K. (1987). An extension of the monocentric urban spatial equilibrium model to a multi-center setting: The case of two center city. Journal of Urban Economics, 21(3), 259-271. doi: 10.1016/0094-1190(87)90001-5

Zheng, X. P. (1991). Metropolitan Spatial Structure and its Determinants: a case study of Tokyo. Urban Studies, 28(1), 87-104. doi: 10.1080/00420989120080061 